\title{
Keck Laser Guide Star Adaptive Optics: Science Verification Results
}

\author{
Antonin H. Bouchez ${ }^{a}$, David Le Mignant ${ }^{a}$, Marcos A. van $\operatorname{Dam}^{b}$, \\ Jason Chin ${ }^{a}$, Scott $\operatorname{Hartman}^{a}$, Erik Johansson ${ }^{a}$, Robert Lafon ${ }^{a}$, \\ Paul Stomski ${ }^{a}$, Douglas Summers ${ }^{a}$, Peter L. Wizinowich ${ }^{a}$ \\ ${ }^{a}$ W. M. Keck Observatory, 65-1120 Mamalahoa Hwy., Kamuela, HI 96743 \\ ${ }^{b}$ Lawrence Livermore National Laboratory, P.O. Box 808, Livermore, CA 94550
}

\begin{abstract}
In this paper we describe the operational strategy and performance of the Keck Observatory laser guidestar adaptive optics system, and showcase some early science verification images and results. Being the first laser guidestar system on an 8-10 m class telescope, the Keck laser guidestar adaptive optics system serves as a testbed for observing techniques and control algorithms. We highlight the techniques used for controlling the telescope focus and wavefront sensor reference centroids, and a wavefront reconstructor optimized for use with an elongated guidestar. We also present the current error budget and performance of the system on tip-tilt stars to magnitude $\mathrm{R}=17$. The capability of the system to perform astronomical observations is finally demonstrated through multi-wavelength imaging of the Egg proto-planetary nebula (CRL 2688).
\end{abstract}

Keywords: adaptive optics, laser guide star

\section{INTRODUCTION}

\subsection{General Introduction}

The Keck laser guidestar (LGS) adaptive optics (AO) system is being developed for the Keck 2 telescope to dramatically expand the region of the sky accessible to high spatial resolution imaging and spectroscopic observations. An overview of the Keck Observatory adaptive optics program is given by Wizinowich et al.. ${ }^{1}$ While the natural guidestar (NGS) adaptive optics systems on both the Keck 1 and Keck 2 telescopes achieve diffractionlimited performance on stars as faint as $\mathrm{V}=12,{ }^{2}$ the LGS AO system is designed to observe any object within $60^{\prime \prime}$ of tip-tilt stars as faint as $\mathrm{V}=18$, opening up $\sim 70 \%$ of the sky to AO-corrected observation. First projection of the sodium-wavelength dye laser occurred in December $2001,{ }^{3}$ and the first laser guidestar-corrected images were achieved in September 2003. ${ }^{1}$ Since that date, monthly on-sky testing and development of the system has gradually improved its performance, efficiency, and reliability. Shared-risk science observations are expected to begin in November 2004.

\subsection{System Description}

The components of the Keck LGS AO system have been previously described, ${ }^{4}$ and we only briefly review them here. The AO bench is located in a clean room on the left Nasmyth platform of the Keck 2 telescope. The LGS AO system consists of all of the NGS AO components, with the addition of a sodium dichroic beamsplitter, an NGS tip-tilt sensor (TTS), and an NGS low-bandwidth wavefront sensor (LBWFS). The sodium dichroic transmits $589 \mathrm{~nm}$ light to the fast wavefront sensor (WFS), while the remaining visible light is directed to the STRAP avalanche photodiode TTS (86\%), the LBWFS (10\%), and an acquisition camera (4\%). The TTS and LBWFS ride on a three-axis positioning stage allowing them to acquire at tip-tilt reference star anywhere within a $60^{\prime \prime}$ radius field. The corrected infrared beam can be directed to either the NIRC2 camera and spectrograph or the NIRSPEC spectrograph. In addition, the OSIRIS OH-suppression integral field spectrograph is due to be installed behind the Keck 2 AO system in September 2004, providing powerful new capabilities to the LGS AO system.

Send correspondence to: abouchez@keck.hawaii.edu; phone (808) 885-7887; fax (808) 885-4464

Presented at SPIE Astronomical Telescopes and Instrumentation Conference, 21-25 June 2004, Glasgow, Scotland, and submitted for inclusion in conference proceedings. 


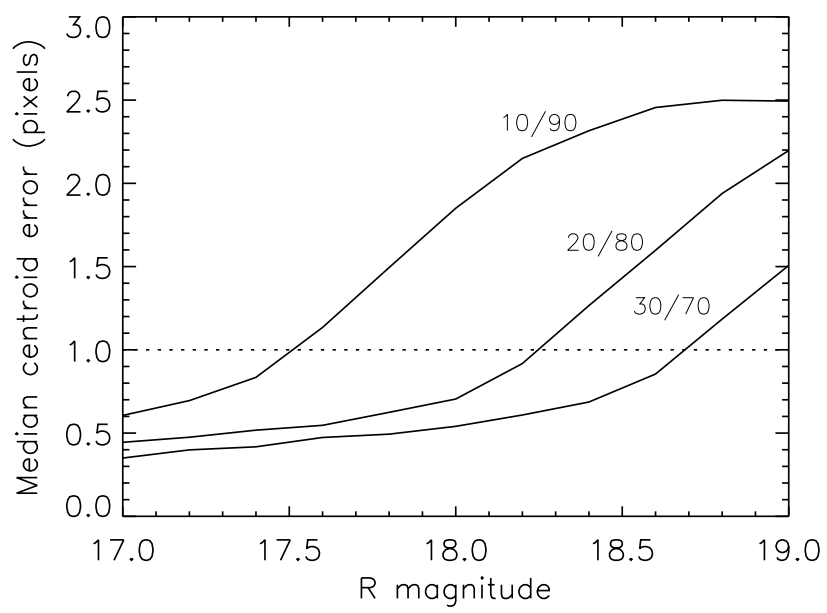

Figure 1. LBWFS centroid determination error versus tip-tilt star magnitude as a function of LBWFS/TTS beamsplitter (reflectivity/transmission) for $60 \mathrm{~s}$ integrations. Focus can be maintained to $0.2 \mathrm{~mm}$ when the median centroid error is below $\sim 1$ pix (dotted line).

In LGS AO operation, the tip-tilt error measured by the TTS on the reference star is sent to the tip-tilt mirror. The WFS, observing the laser guidestar, drives the deformable mirror. Any focus measured by the LBWFS is used to reposition and modify the tracking rate of the WFS focus stage, effectively adjusting the assumed altitude of the atmospheric sodium layer. Higher order aberrations in the reference star wavefront measured with the LBWFS are used to modify the locations to which the WFS spot are driven, eliminating static error induced by the varying elongation of the laser guidestar across the pupil of the telescope. Finally, the mean centroid measured by the WFS is used to drive an uplink tip-tilt (UTT) mirror in the laser launch path, stabilizing the laser guidestar on the wavefront sensor.

Thus, during LGS AO observations, five feedback loops are operating simultaneously. The bandwidth and gain of each must be optimized based on the magnitude and apparent size of the TT reference (TT, focus, and image sharpening loops) and the LGS (UTT and DM loops).

\section{OBSERVING STRATEGY AND CONTROL ALGORITHMS}

\subsection{Focus Control}

Focus control is a key aspect of LGS AO operations. Our requirement for residual focus error is $0.2 \mathrm{~mm}$ on the AO bench, equivalent to $30 \mathrm{~nm}$ of RMS wavefront error. Summers et al. ${ }^{5}$ describe in detail the focus control for Keck LGS AO.

The true focus on the science instrument is maintained by monitoring the average TT reference star wavefront with the LBWFS located on the tip-tilt sensor stage. The subaperture geometry of the LBWFS precisely matches that of the WFS $(20 \times 20$ subapertures $)$. The LBWFS has $16.7 \times 16.7$ pixels per subaperture at $0^{\prime \prime} 148$ pix $^{-1}$. The integration time and CCD binning for the LBWFS are set during operation depending on the star brightness. Figure 1 displays the predicted LBWFS performance for different reflectivity beamsplitters dividing the light between the LBWFS and the TTS. The tip-tilt performance is not shown, but will clearly be reduced as more light is directed to the LBWFS. This analysis indicates that a $20 \%$ reflective beamsplitter will provide optimum performance on the faintest TT stars with which this system will be operated, though we at present use a $10 \%$ reflective beamsplitter.

We have demonstrated $0.4 \mathrm{~mm}$ of focus accuracy for $\mathrm{R}=16.4$ star using $30 \mathrm{~s}$ integrations and $2 \times 2$ binning. On an $\mathrm{R}=17.2$ star, the integration time used was 60 seconds. Such longer integration time reduces the observing efficiency, as it takes about 5 iterations to converge to focus. For TT stars with $\mathrm{R}>15$, we first check the focus and the image sharpening on a nearby brighter star, as described in the acquisition procedure below. 


\subsection{Image Sharpening}

While maintaining the system focused for the science camera is a well-known feature of LGS AO operations, a second less widely acknowledged requirement is the compensation of the quasi-static wavefront error produced by the variable laser spot geometry on the WFS. We call this image sharpening. Image sharpening is an iterative process and can been seen as an additional control loop. We have recently revisited and optimized its implementation on the Keck LGS-AO system so as to increase the sensitivity to faint starlight and minimize noise propagation.

Here we review the various steps involved in this process:

\section{Estimate LBWFS centroids:}

Similarly to the fast WFS, we calibrate the LBWFS: The DM and LBWFS lenslet are registered within 1\% of a subaperture and the LBWFS centroid reference locations are optimized to correct for non-common path aberrations to the NIRC2 science camera. The LBWFS image is processed to subtract the sky background and dark current, and is corrected for flat-fielding and bad pixels. We use a maximum correlation method to locate and extract the spots in the frame and a similar method is used to compute the LBWFS centroids. ${ }^{6}$

\section{Estimate the weight of each subaperture:}

The weight $(w)$ for each subaperture is calculated from the magnitude of the maximum correlation between the observed spots and the reference spots. The weight map is used to identify the apertures which are illuminated as the hexagonal pupil of the telescope rotates.

3 Estimate low order terms $\left(\leq Z_{10}\right)$

An estimate for the 10 lowest order Zernike terms is computed from the centroid map using the expression for the Zernike derivative. ${ }^{7}$ The tip-tilt and focus terms are isolated from the other low terms. The focus term is then converted into millimeters in the focal plane and output to the overall focus manager. ${ }^{5}$

\section{Estimate high order terms}

The low order modes are reconstructed over the entire pupil and subtracted from the measured centroids to derive the higher order component for each illuminated subaperture.

\section{Update WFS centroid references}

Three additional parameters come into play for the integral control loop that provides the new WFS centroid references $\left(C_{i+1}\right)$; an estimate of the WFS spot size $S$ is required to scale accurately the reference centroids, a leak $l$, and a gain factor $k$. The other inputs are $C_{i}^{l o}, C_{i}^{h o}$ the low order and high order components of the WFS reference centroids being used $\left(C_{i}\right), C_{i+1}^{h o}, C_{i+1}^{h o}$ the low order and high order terms from the LBWFS measurement.

$$
C_{i}=l * C_{i}^{l o}+(1-w *(1-l)) * C_{i}^{h o}-k * S *\left(C_{i+1}^{l o}+C_{i+1}^{h o}\right)
$$

Figure 2 illustrates the improvement in performance provided by image sharpening. In this case, the Strehl ratio increased from $8 \%$ to $33 \%$. In position angle (PA) mode, when the field stays fixed on the science camera at a Nasmyth focus, the pupil and LGS elongation pattern rotate on the WFS and LBWFS, and the WFS reference centroids must be updated accordingly. This causes difficulties when observing with faint TT reference stars, as the required WFS centroid corrections may change more rapidly than they can be estimated using the LBWFS. By studying the updates of the WFS reference centroids calculated during previous observing runs, we are investigating on the nature of the aberrations typically seen by the LBWFS. Our plan is to optimize the image sharpening process to work well in PA mode even on faint stars either by predicting these aberrations or by measuring them first on a brighter reference and then implementing modal control for the reference centroids corrections. 

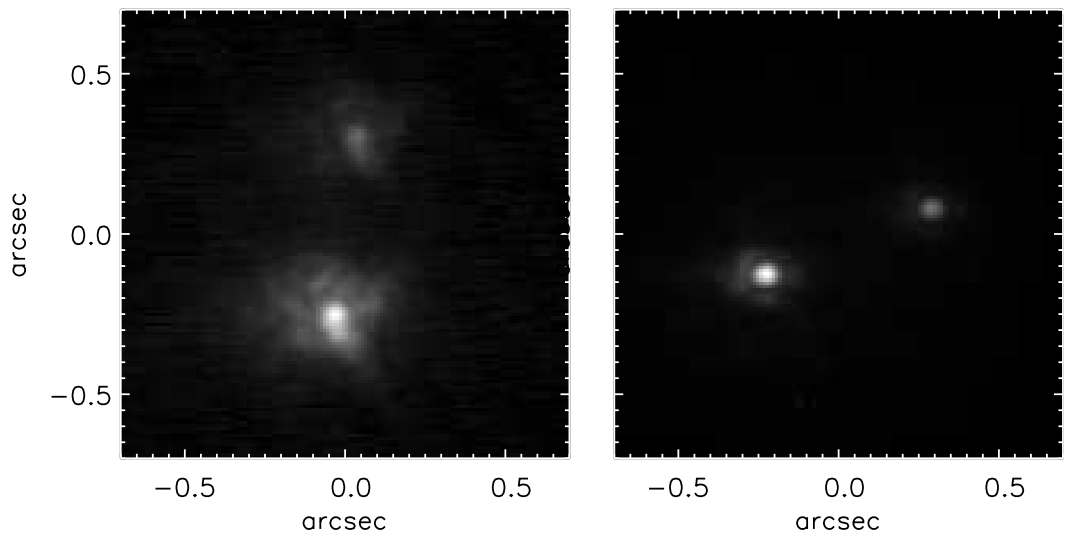

Figure 2. Images of the $0{ }^{\prime \prime} 56$ arcsecond binary star TYC 1467-935-1. The DM was closed on the laser guidestar and the TT loop on the $\mathrm{V}=10.0$ binary. Left: This image was taken after offloading the focus measured with the LBWFS to the DM focus stage, but before proceeding with image sharpening. Strehl is $8 \%$. Right: Image after image sharpening, at a different position angle. Strehl is $33 \%$.

\subsection{Laser Reconstructor}

In addition to generating a quasi-static wavefront error, the varying elongation of the LGS across the pupil also affects the optimum control loop gain for wavefront reconstruction. This gain now becomes a function of pupil position, since regions of the pupil viewing a more extended guidestar should be driven with a higher gain. We have therefore implemented a laser reconstructor which optimizes the gain for each axis of each subaperture.

The laser reconstructor algorithm takes two inputs: the FWHM of the laser spot as seen from near the launch telescope and the maximum elongation observed from the far edge of the pupil. These parameters are a function of laser beam quality, seeing, and the thickness of the atmospheric Na layer, and must be measured nightly from an acquisition camera image of the laser guidestar taken with the primary mirror segments defocused (see center panel of Fig. 3). These dimensions are then used to create an elliptical Gaussian model of the LGS as viewed from each subaperture. These model LGS images are then broadened to account for the charge diffusion in the WFS detector (effectively convolved by a 1". 2 FWHM Gaussian), and the optimum gains for each axis computed. The gains are finally included in a Bayesian reconstructor calculation. ${ }^{8}$ The reconstructor is recomputed whenever the DM loop is closed or the pupil has rotated by more than $0.5^{\circ}$ on the wavefront sensor.

\subsection{Acquisition Procedure}

Both the mean altitude of the atmospheric Na layer and the WFS reference centroids must be determined near the location of a new target before science observations can begin. It may however be more efficient to perform these functions on a nearby bright star, before acquiring the target's fainter tip-tilt reference. In the present configuration of the Keck LGS AO system, with a $10 \%$ reflective beamsplitter directing light to the LBWFS, the limiting magnitude for high-order image sharpening with $60 \mathrm{~s}$ LBWFS integrations is $R \approx 17.5$ (see Fig. 1). However, several iterations (typically $5-10$ ) are required for the image sharpening process to converge. We therefore perform the focus and image sharpening on a nearby bright star for all targets with tip-tilt reference fainter than $R \approx 15$. As noted in Section 2.2, the pattern of WFS centroid offsets determined in this manner will need to be rotated if the pupil angle on the WFS is different at the location of the science target.

The acquisition procedure currently followed for science observations with faint tip-tilt references is therefore as follows:

1. Slew to a bright $(V \approx 10)$ star near the science target.

2. Close TT loop on STRAP TT sensor.

3. Propagate laser. 

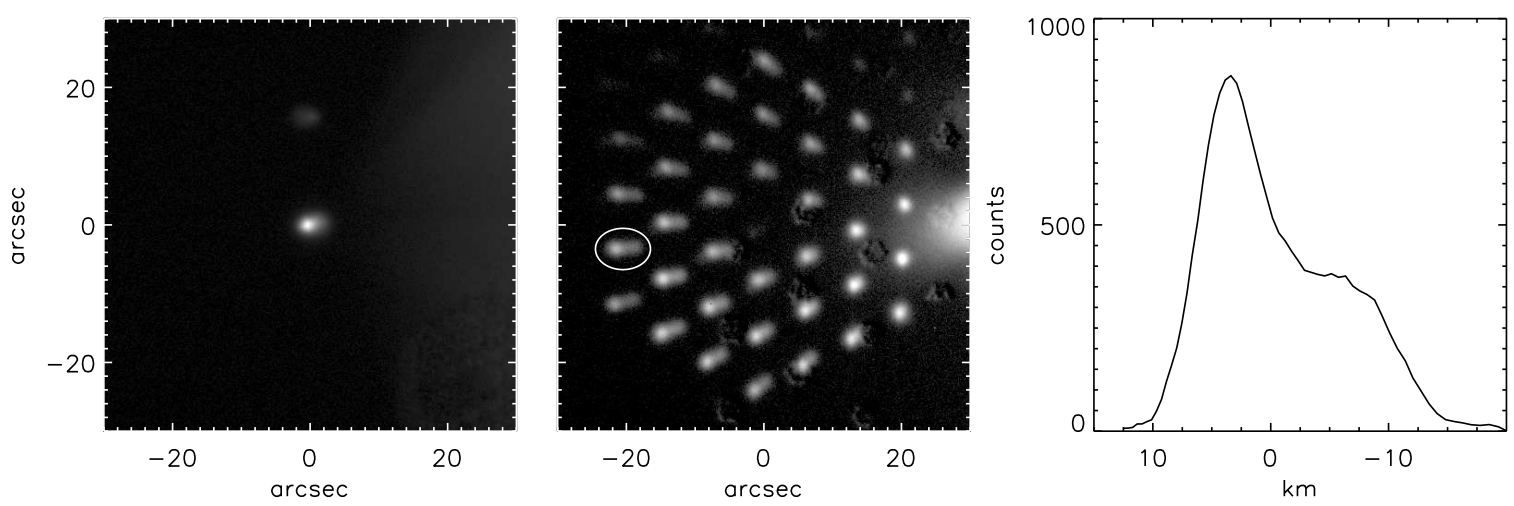

Figure 3. Images of the Keck laser guidestar at zenith taken with the acquisition camera on June 11, 2004 UT. Left: Image of the laser guidestar as seen by the full telescope pupil, with a size of 1 .' $6 \times 22^{\prime \prime} 0 \mathrm{FWHM}$ and magnitude V=9.8. Center: Image of the laser guidestar with the 36 primary mirror segments defocused by $1 \mathrm{~mm}$. Raleigh backscatter can be seen on the right, while the elongation of the laser guidestar increases progressively across the telescope pupil. Right: The relative density structure of the sodium layer derived from the circled image of the laser guidestar.

4. Close UTT loop.

5. Close DM loop on LGS with WFS at predicted Na layer focus.

6. Close LBWFS focus and image sharpening loops, run to convergence.

7. Open loops, shutter laser.

8. Slew to science target.

9. Close TT loop on STRAP TT sensor.

10. Propagate laser.

11. Close UTT loop.

12. Close DM loop on LGS with previously-determined centroid offsets.

13. Offset telescope (and laser guidestar) to science target, keeping the TT reference on the TT sensor.

14. Close LBWFS focus offload loop only.

15. Begin science operations with NIRC2 camera.

This acquisition sequence presently takes $\sim 20$ minutes to perform manually, with approximately half this time spent on the iterative image sharpening process in step 6 . We expect the overall time to be reduced substantially as we optimize the gain of this procedure, and implement general automation tools. These tools will sequence most of the above steps and optimize the integration times, gains, etc., of each feedback loop based on the magnitude of the TT reference star.

\section{PERFORMANCE}

\subsection{Laser Guidestar Performance}

The performance of the LGS AO system depends critically on the power and beam quality of the laser, and the density and structure of the atmospheric sodium layer. The $589 \mathrm{~nm}$ dye laser, ${ }^{3}$ provided by Lawrence Livermore National Labs, is typically operated with an output power of 12-16 W. This generates a laser guidestar whose 
effective magnitude on the wavefront sensor is $8.6<\mathrm{V}<10.2$, with a full-width at half maximum (FWHM) of $1^{\prime \prime} 0-1$.' 4 as seen from the location of the launch telescope. However, the laser guidestar is highly elongated as viewed from more distant regions of the telescope pupil, separated from the launch telescope by up to $11 \mathrm{~m}$. This elongation has been observed to be as great as 3". 4 FWHM, corresponding to a Na layer thickness of $\approx 15 \mathrm{~km}$ (Fig. 3). Such severe elongation leads to strong static aberrations in the resulting LGS AO images, unless image sharpening is performed using the LBWFS.

\subsection{Error Budget}

The current error budget of the Keck LGS AO system, based on observations recorded on 28 February and 11 June 2004, is presented in Table 1. Several of the LGS AO error terms are identical to those in the NGS-AO error budget, and the value of these has been adopted from van Dam et al. ${ }^{2}$ The TT and DM bandwidth and measurement error have been measured using the telemetry returned by these systems while the loops were closed on stars of the magnitude indicated on 11 June 2004. The DM measurement error is surprisingly high due to the weak LGS return on this night, equivalent to only a $\mathrm{V}=10.0$ star. The telescope focus error term was estimated from the standard deviation of the focus measurements returned by the LBWFS, thus assuming no variation in the actual $\mathrm{Na}$ layer altitude over a short $(\sim 10 \mathrm{~min})$ time period.

The focal anisoplanatism error term has not yet been directly measured for the Keck telescopes, and published turbulence profiles ${ }^{9}$ would suggest a higher value $(240 \mathrm{~nm})$. However, we find that a rather small amount of focal anisoplanatism $(155 \mathrm{~nm})$ must be adopted to be consistent with the high Strehl values achieved with LGS AO on bright tip-tilt stars. This result is surprising, and directly measuring the focal anisoplanatism term with the Keck system is a high priority. The "other NGS" and "other LGS" error terms are estimates of the mis-calibration of the WFS, LBWFS, and TT sensors. Finally, the predicted Strehl ratio is computed by the Marechal approximation, which provides an underestimate in the low Strehl regime.

Table 1. Keck NGS and LGS AO error budgets (nm RMS error)

\begin{tabular}{|l|r|r|r|}
\hline & NGS & LGS & LGS \\
\hline R magnitude & 9.0 & 10.0 & 17.0 \\
\hline \hline Camera & 113 & 113 & 113 \\
\hline Fitting & 128 & 128 & 128 \\
\hline Telescope & 60 & 60 & 60 \\
\hline TT bandwidth & 128 & 110 & 270 \\
\hline TT measurement & 0 & 30 & 250 \\
\hline DM bandwidth & 80 & 100 & 100 \\
\hline DM measurement & 80 & 275 & 275 \\
\hline Focal anisoplanatism & 0 & 155 & 155 \\
\hline Telescope focus & 0 & 35 & 90 \\
\hline Other NGS & 105 & 105 & 105 \\
\hline Other LGS & 0 & 100 & 100 \\
\hline \hline Total wavefront error & 270 & 421 & 550 \\
\hline Predicted Strehl & 0.52 & 0.20 & 0.06 \\
\hline Measured Strehl & 0.55 & 0.33 & 0.13 \\
\hline
\end{tabular}

This error budget provides a clear indication of which aspects of the LGS AO system currently limit our performance and must be modified or further optimized. We expect to be dominated by TT measurement and bandwidth error when using faint TT stars, so the equally high DM measurement error indicates that increasing 

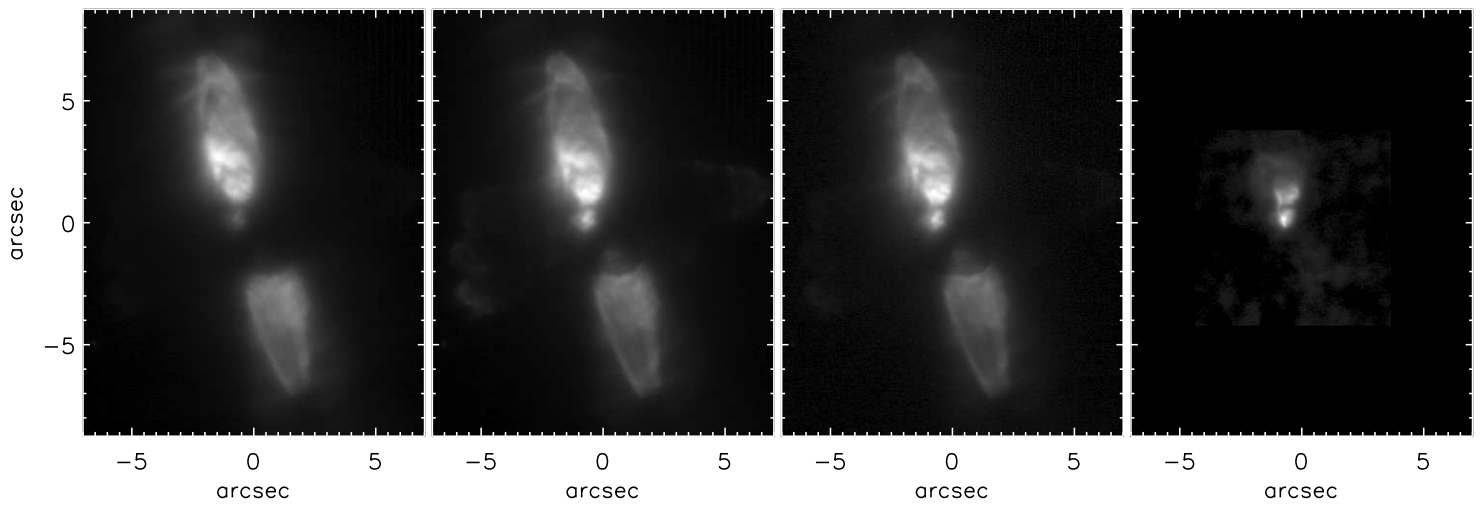

Figure 4. Mean images of the Egg Nebula in $\mathrm{H}, \mathrm{K}^{\prime}, \mathrm{H}_{2}(2-1)$, and $\mathrm{L}^{\prime}$ filters. The images have been registered to one another by cross-correlation, and the origin of the axes fixed at the predicted location of the hidden central AGB star. ${ }^{10}$

the return from the LGS must be one of our highest development priorities. A new dye pump should soon allow us to run the laser at up to $50 \%$ higher power, resolving this issue. However, a substantial performance increase on faint tip-tilt stars will also require an improvement in the TT performance. This will surely remain the limiting error term for some time to come.

\section{OBSERVATIONS OF THE EGG NEBULA}

Apart from the initial demonstration of the LGS AO system on the HK Tau young star system, ${ }^{1}$ the first target of the LGS AO science verification program was the proto-planetary Egg Nebula (CRL 2688, see Fig. 4). The Egg Nebula is the best-studied proto-planetary nebula, the short-lived transitional phase through which 1-8 $\mathrm{M}_{\odot}$ stars pass between slow evolution on the active giant branch (AGB) and their final destiny as white dwarfs. This is a period of rapid mass loss, and of transformation from the spherically-symmetric envelopes of AGB stars to the complex and beautiful shapes of planetary nebulae.

The Egg Nebula was observed with the Keck LGS AO system between 12:33 and 13:56 UT on 11 June 2004. The tip-tilt reference star used was USNO-1B 1266-0470099, an $\mathrm{R}=14.3$ star 24 ". 9 south-east of the nebula. This star was sufficiently bright to allow the TT sensor to be run at its full frame rate of $1 \mathrm{MHz}$ and the LBWFS to correct both focus and static high-order aberrations using $30 \mathrm{~s}$ integrations. However, due to the rather low laser power output that night, the LGS magnitude was only V=10.0, limiting the WFS frame rate to $250 \mathrm{~Hz}$. Seeing was also worse than average, $00^{\prime \prime} 83$ at $2.1 \mu \mathrm{m}$.

Images of the Egg Nebula were taken in $\mathrm{H}, \mathrm{K}^{\prime}, \mathrm{H}_{2} S(1) \nu=2-1$, and $\mathrm{L}^{\prime}$ filters, with sky observations in each filter gathered by opening the loops and offsetting $60^{\prime \prime}$ from the target in several directions. The raw images were sorted by the energy near the diffraction limit in their power spectra, and only the best $50 \%$ retained. The median of the sky frames was subtracted from each of these images, which were then divided by twilight flats. Finally, the individual images were co-aligned to the nearest pixel and averaged to derive a final mean image of the nebula in each of the four filters, displayed in Fig. 4. The total integration time for the mean images is $120 \mathrm{~s}$ in $\mathrm{H}, 360 \mathrm{~s}$ in $\mathrm{K}^{\prime}, 240 \mathrm{~s}$ in $\mathrm{H}_{2}(2-1)$, and $144 \mathrm{~s}$ in $\mathrm{L}^{\prime}$.

A comparison to one of the best Hubble Space Telescope (HST) Near Infrared Camera and Multi-object Spectrograph (NICMOS) image of the Egg Nebula ${ }^{11}$ is shown in Fig. 5. The HST image is a 1215 s integration with an $\mathrm{H}_{2} S(1) \nu=1-0$ filter, through which the complex equatorial lobes are most clearly defined. The Keck 

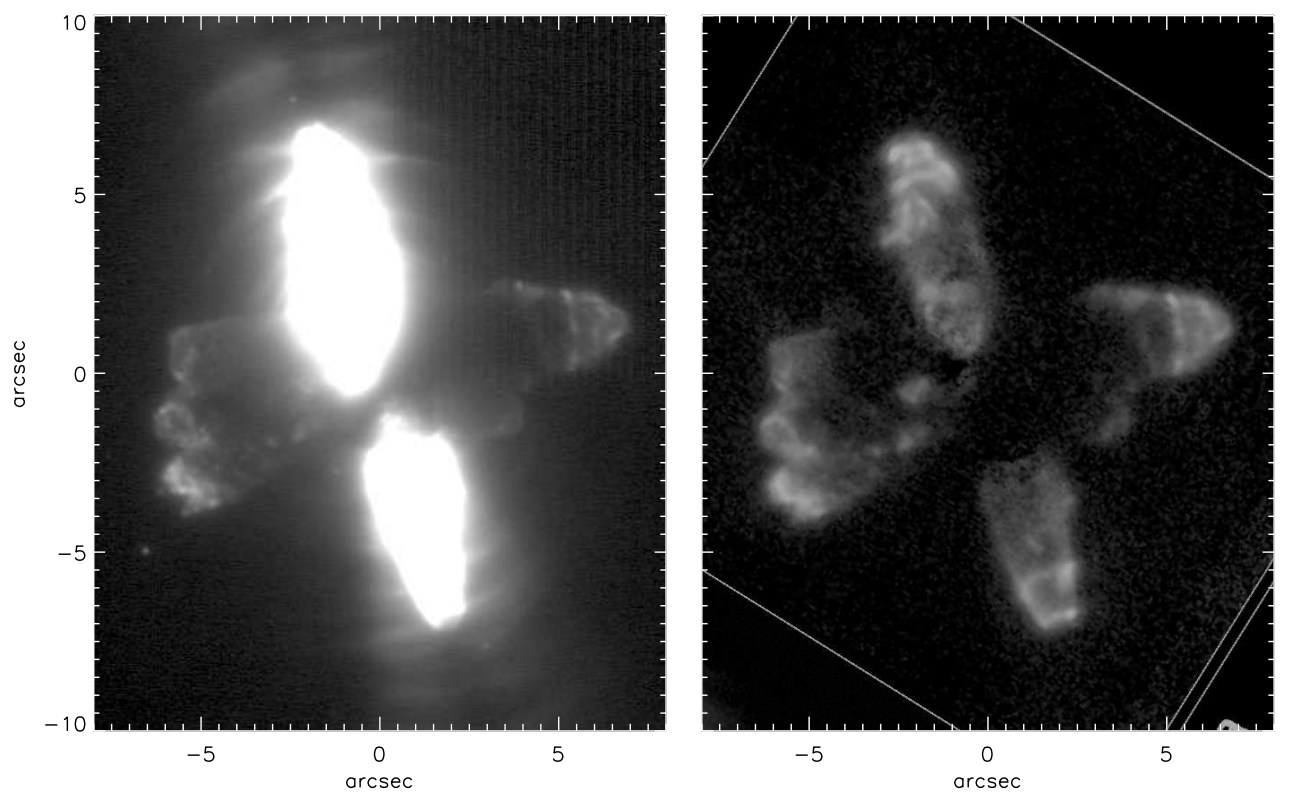

Figure 5. Comparison of the Keck LGS AO K' image and an HST NICMOS image in a narrow-band $\mathrm{H}_{2} S(1) \nu=1-0$ filter which highlights the shocked emission emanating from the cavity walls. The LGS AO image has been stretched to highlight details in the equatorial lobes, clearly illustrating the higher angular resolution and dynamic range of this image. The shocked $\mathrm{H}_{2}$ emission is resolved into clumps and knots, and can be traced closer in to the central star. The FWHM of the faint background star at $\left(-6 .{ }^{\prime \prime} 5,-5 .{ }^{\prime \prime} 0\right)$ in the Keck $\mathrm{K}^{\prime}$ image is $0 .{ }^{\prime \prime} 14$.

images clearly have both higher angular resolution and greater dynamic range, an impressive demonstration of the power of LGS AO on large ground-based telescopes.

Based HST and previous seeing-limited observations, Sahai et al. ${ }^{11,12}$ suggested a physical model of the Egg Nebula which appears generally well-supported by these higher resolution images. The multiple bright lobes seen in the near-IR images are most likely cavities blown into a spherically-symmetric and optically thin dust shell by collimated winds originating from the central AGB star, which remains hidden in a flattened dust cocoon. Their walls are illuminated by scattered light from the hidden central AGB star (in the case of the bright NW-SE polar lobes), and by shock-heated $\mathrm{H}_{2}$ and possibly other molecular emission (the fainter equatorial lobes). The "searchlight beams" extending to far greater distances are created by the scattering of shorter-wavelength light escaping from the central cocoon through annular holes around the polar axis, presumably carved by precessing, highly collimated winds.

One of the more puzzling questions is the nature of the bright knot just south of the northern lobe, visible in all four panels of Fig. 4. This feature, not seen at wavelengths shorter than $1.6 \mu \mathrm{m},{ }^{11}$ is $0{ }^{\prime \prime} 55$ offset from the location of the AGB star predicted from the polarization symmetry of the northern and southern lobes. ${ }^{10}$ Based on its marginally-resolved appearance in HST NICMOS images and its low polarization, Sahai et al. ${ }^{11}$ suggest that it is a lower-mass companion star, imbedded in the near side of the dust cocoon and thus surrounded by a halo of scattered light. The high resolution of the Keck LGS AO 2.0 and $3.5 \mu \mathrm{m}$ images provides a surprising new insight into the nature of this feature.

A more detailed view of the central few arcsecond region of the Egg Nebula viewed through the $\mathrm{H}_{2}(2-1)$ and $\mathrm{L}^{\prime}$ filters is shown in Fig. 6. The $\mathrm{L}^{\prime}$ image, while having lower dynamic range than previous seeing-limited imaging, ${ }^{13}$ reveals the detailed structure of the bright knot at $\left(00^{\prime \prime} 56 \mathrm{~W}, 0{ }^{\prime \prime} 16 \mathrm{~N}\right)$, clearly revealing that it is actually the apex of the northern lobe. It is connected to the brighter (at $\lambda<2 \mu \mathrm{m}$ ) regions of the northern lobe by a sinuous but unbroken lane of scattered light, hidden from view at shorter wavelengths by a cloud of foreground dust. 

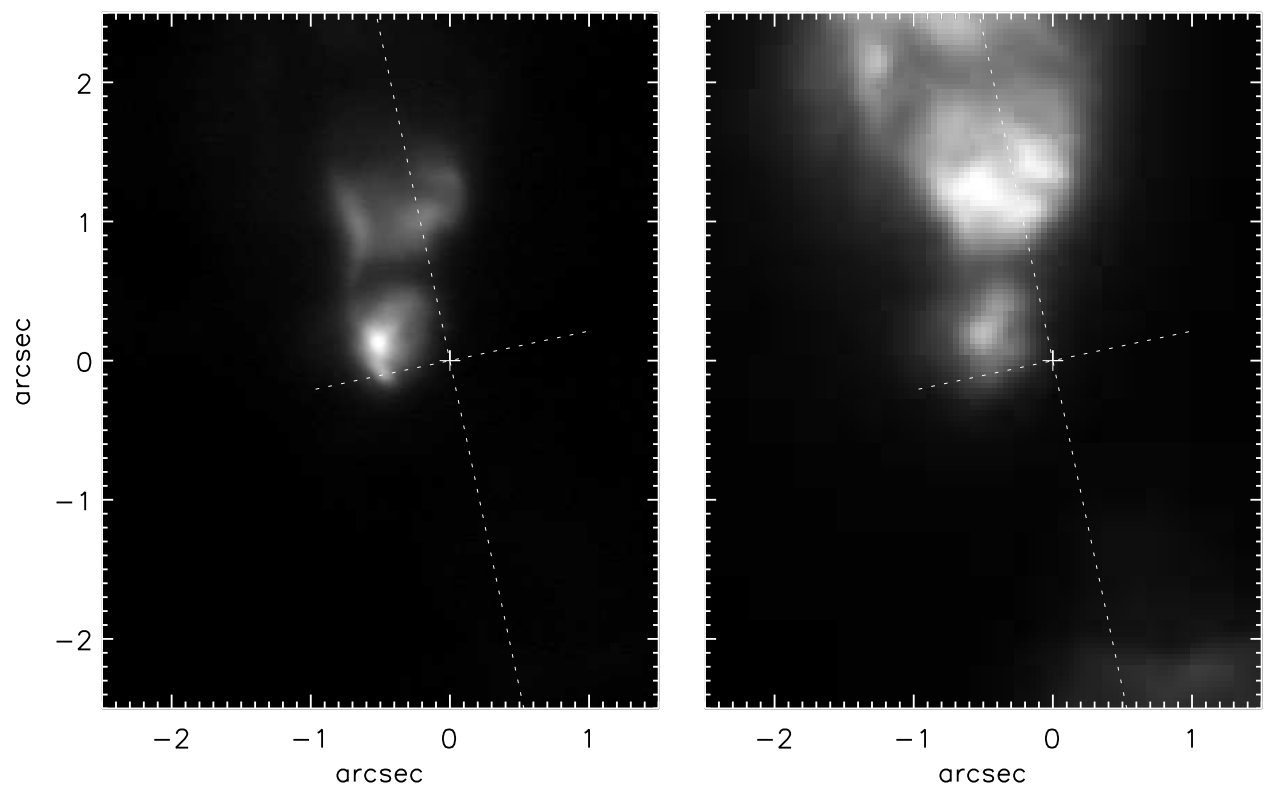

Figure 6. A detailed view of the central region of the Egg Nebula, in $\mathrm{L}^{\prime}$ (left) and $\mathrm{H}_{2}(2-1)$ (right). The predicted location of the central AGB star, and the polar and equatorial axes, are indicated with long and short dotted lines. ${ }^{10}$ Previous seeing-limited $\mathrm{L}^{\prime}$ observations did not resolve the connection between the bright knot at $(0 . .56 \mathrm{~W}, 0$.' $16 \mathrm{~N})$ and the base of the north lobe. ${ }^{13}$ This connection reveals that the bright knot is in fact the apex of the northern lobe cavity, seen only at longer wavelength due to strong extinction within the dust cocoon. We suggest that the true location of the central AGB star in fact just south of this knot, and the polarization axes are biased due to multiple scattering of light within the cavities.

Based on the morphology the inner regions of the Egg Nebula revealed by the Keck LGS AO L' image, we suggest that the central AGB star is in fact located just south of the bight knot, rather than at the apparent point of polarization symmetry. The $\mathrm{L}^{\prime}$ image clearly reveals in scattered light the corkscrew-like walls of the innermost northern cavity, consistent with it being carved out of a cloud of dust by a collimated and wildly precessing wind. Given the continuity and narrowing of the cavity walls, the star giving rise to these winds must undoubtedly be located at the apex of this feature. This is $\sim 00^{\prime \prime} 5$ distant from the location suggested by Weintraub et al., ${ }^{10}$ and not along the apparent polarization axis of the nebula.

The observed polarization of the polar lobes can be explained by the sinuous shape of the northern cavity apex, and high optical depth of the inner regions of the dust cocoon. Photons detected from the more distant regions of the northern and southern lobes may have been scattered multiple times at the cavity's walls, their polarization preserving only a record of their plane of propagation immediately prior to the final scattering event. It thus appears likely that the AGB star is in fact located just south of the bright knot, at the apex of two narrow, sinuous cavities out of which only multiply-scattered light emerges. Future measurements of the polarization of the innermost region at $\lambda>2 \mu \mathrm{m}$ should be able to confirm or refute this hypothesis.

\section{CONCLUSIONS}

After over a decade of planning and development, the Keck laser guidestar adaptive optics system is now operational and revealing the faint universe in unparalleled detail. Many of the lessons learned optimizing this first LGS AO system on an 8-10 m telescope have been presented in this paper, as has an analysis of the sources of wavefront error which limit the present performance. While further optimization remains to be performed, the correction already demonstrated suggest that focal anisoplanatism will not be the dominant error term. The observations of the proto-planetary Egg Nebula presented here provide a taste of the spectacular high-resolution science possible with LGS AO. 


\section{ACKNOWLEDGMENTS}

We would like to thank Joel Aycock, Julie Rivera, Chuck Sorenson, Terry Stickel, Cynthia Wilburn, and Ian Lynn for able assistance during observing runs. The data presented herein were obtained at the W. M. Keck Observatory, which is operated as a scientific partnership among the California Institute of Technology, the University of California and the National Aeronautics and Space Administration. The Observatory was made possible by the generous financial support of the W. M. Keck Foundation. The authors wish to recognize and acknowledge the significant cultural role and reverence that the summit of Mauna Kea has always had within the Hawaiian community. We are most fortunate to have the opportunity to conduct observations from this mountain.

\section{REFERENCES}

1. P. L. Wizinowich, D. Le Mignant, A. H. Bouchez, J. Chin, A. R. Contos, S. Hartman, E. Johansson, R. Lafon, C. Neyman, P. J. Stomski, D. Summers, and M. van Dam, "Adaptive Optics Developments at Keck Observatory," 2004. Submitted to Proceedings of the SPIE.

2. M. A. van Dam and B. A. Macintosh, "Characterization of adaptive optics at Keck Observatory," in Astronomical Adaptive Optics Systems and Applications. Edited by Tyson, Robert K.; Lloyd-Hart, Michael. Proceedings of the SPIE, Volume 5169, pp. 1-10 (2003)., pp. 1-10, Dec. 2003.

3. D. M. Pennington, C. G. Brown, P. M. Danforth, and H. E. Jones, "Current performance and status of the Keck Observatory guide star laser system," in Adaptive Optical System Technologies II. Edited by Wizinowich, Peter L.; Bonaccini, Domenico. Proceedings of the SPIE, Volume 4839, Addendum (2003)., Feb. 2003.

4. A. R. Contos, P. L. Wizinowich, S. K. Hartman, D. Le Mignant, C. R. Neyman, P. J. Stomski, and D. Summers, "Laser guide star adaptive optics at the Keck Observatory," in Adaptive Optical System Technologies II. Edited by Wizinowich, Peter L.; Bonaccini, Domenico. Proceedings of the SPIE, Volume 4839, pp. 370-380 (2003)., pp. 370-380, Feb. 2003.

5. D. Summers, A. H. Bouchez, J. Chin, A. R. Contos, S. Hartman, E. Johansson, R. Lafon, D. Le Mignant, P. J. Stomski, M. van Dam, and P. L. Wizinowich, "Focus and pointing adjustments necessary for laser guide star adaptive optics at the W.M. Keck Observatory," 2004. Submitted to Proceedings of the SPIE.

6. L. A. Poyneer, "Advanced techniques for Fourier transform wavefront reconstruction," in Adaptive Optical System Technologies II. Edited by Wizinowich, Peter L.; Bonaccini, Domenico. Proceedings of the SPIE, Volume 4839, pp. 1023-1034 (2003)., pp. 1023-1034, Feb. 2003.

7. R. J. Noll, "Phase estimates from slope-type wave-front sensors (T)," Optical Society of America Journal 68, pp. 139-+, 1978.

8. M. A. van Dam and B. A. Macintosh, "Characterization of adaptive optics at Keck Observatory: part II," 2004. Submitted to Proceedings of the SPIE.

9. R. Racine and B. L. Ellerbroek, "Profiles of nighttime turbulence above Mauna Kea and isoplanatism extension in adaptive optics," in Proc. SPIE Vol. 2534, p. 248-257, Adaptive Optical Systems and Applications, Robert K. Tyson; Robert Q. Fugate; Eds., pp. 248-257, Aug. 1995.

10. D. A. Weintraub, J. H. Kastner, D. C. Hines, and R. Sahai, "Pinpointing the Position of the Post-Asymptotic Giant Branch Star at the Core of RAFGL 2688 Using Polarimetric Imaging with NICMOS," Astroph. J. 531, pp. 401-406, Mar. 2000.

11. R. Sahai, D. C. Hines, J. H. Kastner, D. A. Weintraub, J. T. Trauger, M. J. Rieke, R. I. Thompson, and G. Schneider, "The Structure of the Prototype Bipolar Protoplanetary Nebula CRL 2688 (Egg Nebula): Broadband, Polarimetric, and H 2 Line Imaging with NICMOS on the Hubble Space Telescope," Astroph. J. Lett. 492, pp. L163+, Jan. 1998.

12. R. Sahai, J. T. Trauger, A. M. Watson, K. R. Stapelfeldt, J. J. Hester, C. J. Burrows, G. E. Ballister, J. T. Clarke, D. Crisp, R. W. Evans, J. S. Gallagher, R. E. Griffiths, J. G. Hoessel, J. A. Holtzman, J. R. Mould, P. A. Scowen, and J. A. Westphal, "Imaging of the EGG Nebula (CRL 2688) with WFPC2/HST: A History of AGB/Post-AGB Giant Branch Mass Loss," Astroph. J. 493, pp. 301-+, Jan. 1998.

13. M. Goto, N. Kobayashi, H. Terada, and A. T. Tokunaga, "Imaging and Spatially Resolved Spectroscopy of AFGL 2688 in the Thermal Infrared Region," Astroph. J. 572, pp. 276-287, June 2002. 